\title{
GESTÃO DE DESIGN NA SAÚDE HOSPITALAR: ESTUDO DE CASO NO INSTITUTO DE PSIQUIATRIA DE SANTA CATARINA (IPQ-SC)
}

Giselle Schmidt Alves Díaz Merino, Dra

Universidade Federal de Santa Catarina

Universidade do Estado de Santa Catarina

gisellemerino@gmail.com

Renata Hinnig

Universidade Federal de Santa Catarina ${ }^{1}$

renatahinnig@gmail.com

Susana Domenech, Dra

Universidade do Estado de Santa Catarina

scdomenech@gmail.com

Eugenio Andrés Díaz Merino, PhD

Universidade Federal de Santa Catarina

eugenio.merino@ufsc.br

Resumo: A gestão de design busca gerir pessoas, projetos, processos e procedimentos que resultam no desenvolvimento de produtos, ambientes e experiências. Pensar no design no contexto da medicina e dos cuidados com a saúde é algo relativamente novo. A presente pesquisa demonstra as contribuições da gestão de design aplicada a projetos de design que visam melhorar a qualidade dos serviços de saúde prestados aos pacientes, bem como a melhoria das condições de trabalho dos funcionários no Instituto de Psiquiatria de Santa Catarina (IPq-SC). Trata-se de um estudo de caso, onde foi utilizada a metodologia Guia de Orientação para Desenvolvimento de Projetos (GODP) para gestão de design. Como contribuições da gestão de design no contexto da área da saúde, pode-se citar a capacidade de coordenar projetos com equipes multidisciplinares, considerando os objetivos da organização.

Palavras-chave: Gestão de Design, Design Estratégico, Serviços de Saúde, Psiquiatria

Abstract: Design management seeks to manage people, projects, processes and procedures which result in the development of products, environments and experiences. Reflecting on design in the context of medicine and health care is relatively new. This research demonstrates the contributions of design management applied to design projects which aimed to improve the

\footnotetext{
${ }^{1}$ Bolsista CAPES pela Rede de Pesquisa e Desenvolvimento em Tecnologia Assistiva (RPDTA).
} 
quality of health care services and the improvement of working conditions for the employees in the Santa Catarina Institute of Psychiatry (IPq-SC). It is a case study where the Guidance Methodology for Project Development (GODP) has been used for the design management. As a result, one can cite that the design management contributes to coordinate projects with multidisciplinary teams, considering the organization's objectives.

Keywords: Design Management, Strategic Design, Health Care Services, Psychiatry.

\section{INTRODUÇÃO}

Para que o design possa ser aplicado de maneira mais eficiente e eficaz nas organizações a gestão de design busca gerir pessoas, projetos, processos e procedimentos que resultam na criação de produtos, ambientes e experiências (BEST, 2012). Esse gerenciamento envolve a gestão das relações entre diferentes disciplinas e diferentes profissionais envolvidos nos projetos de design (BEST, 2012).

Segundo Park (2015, p. 63, tradução nossa) "pensar no design no contexto da medicina e dos cuidados com a saúde é algo relativamente novo". Para o autor (PARK 2015), uma das primeiras iniciativas do uso do design no contexto da saúde ocorreu em 1960, com o projeto do King's Fund Bed, considerado um protótipo de todos os leitos utilizados atualmente em hospitais. Desde então, essa aproximação entre o design e a área da saúde vem crescendo, e atualmente pode-se visualizar algumas da áreas onde o design vem fazendo contribuições significativas: arquitetura de ambientes internos e externos de hospitais, desenvolvimento de aplicativos de $e$ health, projeto de dispositivos e aparelhos para serem utilizados por médicos, enfermeiros ou pacientes, além de projetos que procuram envolver diversos profissionais da área da saúde e pacientes, como as iniciativas que incluem co-design e participatory health care design (design participativo na área da saúde) (PARK, 2015).

Os exemplos acima demonstram que o design pode oferecer importantes contribuições para a área da saúde, buscando a melhoria e inovação em produtos e serviços com o intuito de oferecer soluções que possuem como foco as necessidades dos usuários.

A complexidade que existe nos serviços de saúde, por abrigarem interesses variados de pacientes, profissionais de saúde (médicos, enfermeiros, etc.), governos, entre outros, requer que qualquer intervenção feita nesse sistema considere as diferentes perspectivas dos diversos atores envolvidos nesse processo (JONES, 2013). O design, em sua prática projetual, possui como uma das suas atividades principais lidar com essa complexidade (STROPKAY; SIEDZIK, 2012).

A pesquisa, quando foca na experiência dos pacientes e trabalhadores da saúde, vai de encontro ao Programa Nacional de Humanização do Atendimento Hospitalar, lançado em 2001 pelo Ministério da Saúde, e que em 2003 transformou-se em política pública: a Política Nacional de Humanização (BRASIL, 2001a). Também se encontra alinhada às políticas do Ministério do Trabalho e Emprego, que buscam a promoção da saúde e a melhoria da qualidade de vida do trabalhador, bem como a prevenção de acidentes e de danos à saúde relacionados ao trabalho (PRESIDÊNCIA DA 
REPÚBLICA, 2011).

Considerando o contexto apresentado, pretende-se por meio de um estudo de caso demonstrar as contribuições da aplicação da gestão de design utilizando a metodologia Guia de Orientação para Desenvolvimento de Projetos - GODP (MERINO, 2014) em projetos de design que visam melhorar a qualidade dos serviços de saúde prestados aos pacientes, bem como a melhoria das condições de trabalho dos funcionários no Instituto de Psiquiatria de Santa Catarina (IPq-SC), objeto de estudo dessa pesquisa.

A pesquisa faz parte de 3 projetos $^{2}$ que encontram-se em desenvolvimento por meio do Núcleo de Gestão de Design e Laboratório de Design e Usabilidade (NGD/LDU) ${ }^{3}$ da Universidade Federal de Santa Catarina (UFSC).

Após a presente introdução, serão conceituados gestão de design e saúde do paciente e trabalhador no ambiente hospitalar. Em seguida, serão descritos os procedimentos metodológicos da pesquisa. Na sequência será apresentado o estudo de caso, e nas considerações finais serão apontadas as contribuições da gestão de design para os projetos de design aplicados nesse estudo. A estrutura da pesquisa pode ser visualizada na figura 1.

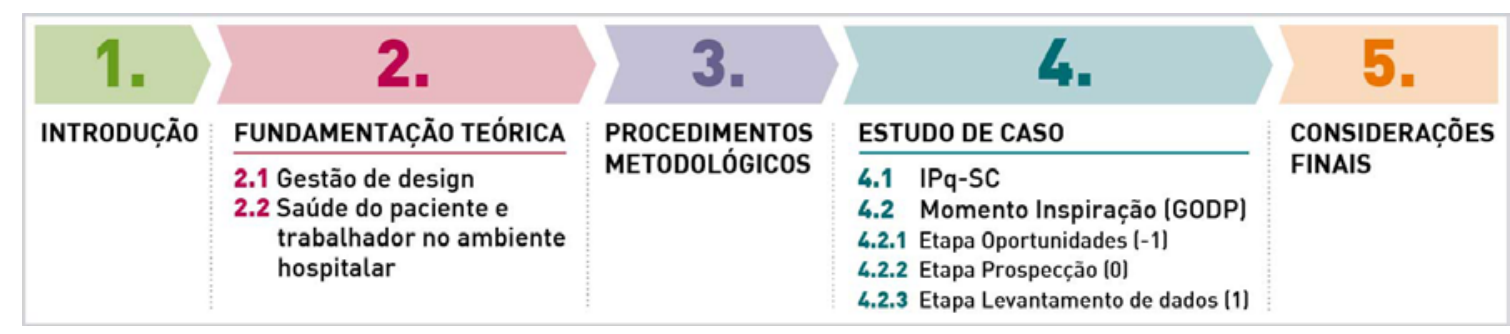

Figura 1 - Estrutura da pesquisa.

Fonte: Elaborado pelos autores, com base na pesquisa realizada.

\section{FUNDAMENTAÇÃO TEÓRICA}

\subsection{Gestão de design}

A gestão de design trata do conjunto de atividades de diagnóstico, coordenação, negociação e design que pode ser feita tanto por equipe interna na organização, como por consultoria externa (AVENDÃNO, 2005). Cabe a gestão de design determinar como reunir pessoas, projetos e processos de forma interdisciplinar e colaborativa, considerando que esses fatores estão inseridos em um contexto empresarial, social, político e ambiental mais amplo (BEST, 2012). A gestão de design é a arte e a ciência que melhora a eficácia do design pois contribui para aumentar a colaboração e a sinergia entre design e negócios (DMI, 2016).

As atribuições da gestão de design apontadas acima por Best (2012), vêm de encontro ao que o Centro Português de Design (1997) cita como responsabilidades do

\footnotetext{
${ }^{2}$ Os três projetos mencionados são: 1) Edital PROEX (MEC-SESu) - Psiquiatria em análise: saúde do paciente às questões da saúde do trabalhador; 2) Edital CAPES - RPDTA: ações integradas entre engenharia mecânica e design; 3) Edital Universal CNPq - Design e Saúde: contribuição do desenho industrial na reabilitação de pacientes e trabalhadores inseridos em ambientes psiquiátricos.

${ }^{3}$ O NGD/LDU atua desde 2000 tendo como foco investigar, aplicar e disseminar o design e a ergonomia como ferramentas estratégicas para as organizações, tendo como pilares de sustentação a competitividade, a diferenciação e a sustentabilidade, aplicada a projetos, produtos e serviços com ênfase no Design Universal - Inclusivo (NGD/LDU, 2016).
} 
gestor de design: buscar o alinhamento com as estratégias da empresa, avaliar o problema e os recursos necessários, planificar o projeto, selecionar equipe e especialistas externos, definir a forma de atuação, organizar o processo (procedimentos, fases, níveis de decisão e comunicações), levantar a documentação necessária, acompanhar e controlar o processo, realizar avaliações parciais e uma avaliação final.

Quanto a introdução da gestão de design nas organizações, essa deve feita de maneira gradativa e responsável (MOZOTA, 2003), ou seja, em etapas e por meio de uma sequência de vários projetos, para que possa ser inserida de maneira espontânea e que ao longo do tempo ajude a formar uma cultura de Design na organização. (MARTINS; MERINO, 2011). Moreira et al. (2016) defendem que ao iniciar um projeto de design seja feita uma etapa de sensibilização junto a organização para nivelar os conhecimentos acerca da gestão de design. Essa etapa torna-se importante devido ao desconhecimento que existe dos benefícios do design e consequentemente de sua gestão (MOREIRA et al., 2016).

Entende-se que a gestão de design busca fazer com que o design se insira nas organizações de maneira responsável e coordenada, procurando compreender as diversas variáveis (externas e internas) que irão influenciar os projetos de design, bem como considerar o design deve atuar de acordo com os objetivos estratégicos da organização onde está inserido.

\subsection{Saúde do paciente e trabalhador no ambiente hospitalar}

A Organização Mundial de Saúde - OMS (WHO, 2016a) define saúde não apenas como a ausência de doença, mas como a situação de perfeito bem-estar físico, mental e social. Esse conceito de saúde é bastante amplo e compreende tanto ações e serviços que visam a prevenção de doenças como serviços que buscam tratar as doenças. Nessa pesquisa, a saúde possui essas duas abordagens, pois tem foco na saúde de pacientes, que se enquadram no conceito de cura das doenças, como a saúde dos trabalhadores, que engloba a prevenção de doenças.

Tendo como objeto de estudo de caso, um ambiente hospitalar, serão abordados a seguir algumas das peculiaridades referentes a esse ambiente.

Hospitais são instituições de saúde que possuem uma equipe profissional organizada e instalações para receber pacientes, com a finalidade de entregar serviços médicos, de enfermagem e outros serviços relacionados em tempo integral (WHO, 2016b). O ambiente hospitalar é considerado rico, estimulante e heterogêneo, ao mesmo tempo em que compreende atividades insalubres, penosas e difíceis para os diversos trabalhadores envolvidos nesse contexto (médicos, enfermeiros, fisioterapeutas, psicólogos, etc.) (LIMA JúNIOR; ÉSTHER, 2001).

Com relação as características próprias dos serviços de saúde psiquiátricos, cabe ressaltar que existem diversos desafios a serem superados nessa área no Brasil, pelas mudanças que vem ocorrendo decorrentes da Reforma Psiquiátrica ${ }^{4}$, e que buscam humanizar a assistência psiquiátrica, com ênfase na reabilitação ativa em detrimento da custódia e da segregação dos pacientes (MACIEL, 2012).

Acredita-se que o design pode contribuir para a área da saúde e no ambiente

\footnotetext{
${ }^{4}$ Consolidada a partir da Lei no 10.216 (BRASIL, 2001b), a Reforma Psiquiátrica questiona as internações por longos períodos, propondo a extinção e a substituição progressiva dos manicômios por novas modalidades de atendimento (MACIEL, 2012).
} 
hospitalar. Lucio e Paschoarelli (2009) argumentam que no âmbito hospitalar, a integração entre as diversas áreas de conhecimento como a acessibilidade, a antropometria, o Design Ergonômico, o Design Universal, a Ergonomia e a Usabilidade, corroboram para o emprego de soluções mais condizentes com as reais necessidades dos usuários, permitindo contemplar as diversas potencialidades, as quais não seriam adequadamente atendidas sob uma única ótica. A Ergonomia e a Usabilidade são consideradas fontes ricas de conceitos e métodos que permitem a concepção ou a correção de projetos de forma harmoniosa com o sistema de saúde (SERRANHEIRA, UVA; SOUSA, 2010; REID et al, 2005).

Sendo assim, acredita-se que a aproximação da área do design com a saúde, pode ajudar na melhoria dos serviços na área da psiquiatria, tanto com foco nos pacientes, como nos trabalhadores do ambiente hospitalar.

\section{PROCEDIMENTOS METODOLÓGICOS}

Do ponto de vista de sua natureza a pesquisa caracteriza-se como aplicada. Quanto a forma de abordagem é qualitativa e possui objetivos exploratórios (SILVA, MENEZES, 2005). Com base nos procedimentos técnicos, a pesquisa classifica-se como estudo de caso, pois compreende " $[. .$.$] um estudo empírico que investiga um$ fenômeno atual dentro de seu contexto de realidade" (GIL, 2007, p. 73).

No estudo de caso, foi utilizado como metodologia para a gestão de design o Guia de Orientação para Desenvolvimento de Projetos (GODP). Essa metodologia busca organizar e oferecer uma sequência de ações que permitam com que o design de produtos e serviços seja concebido de forma consciente, considerando o maior número de aspectos e respondendo de forma mais assertiva e consistente aos objetivos fixados para a prática projetual (MERINO, 2014). O GODP (figura 2) compreende 3 momentos (Inspiração, Ideação e Implementação), que se decompõem em 8 etapas.

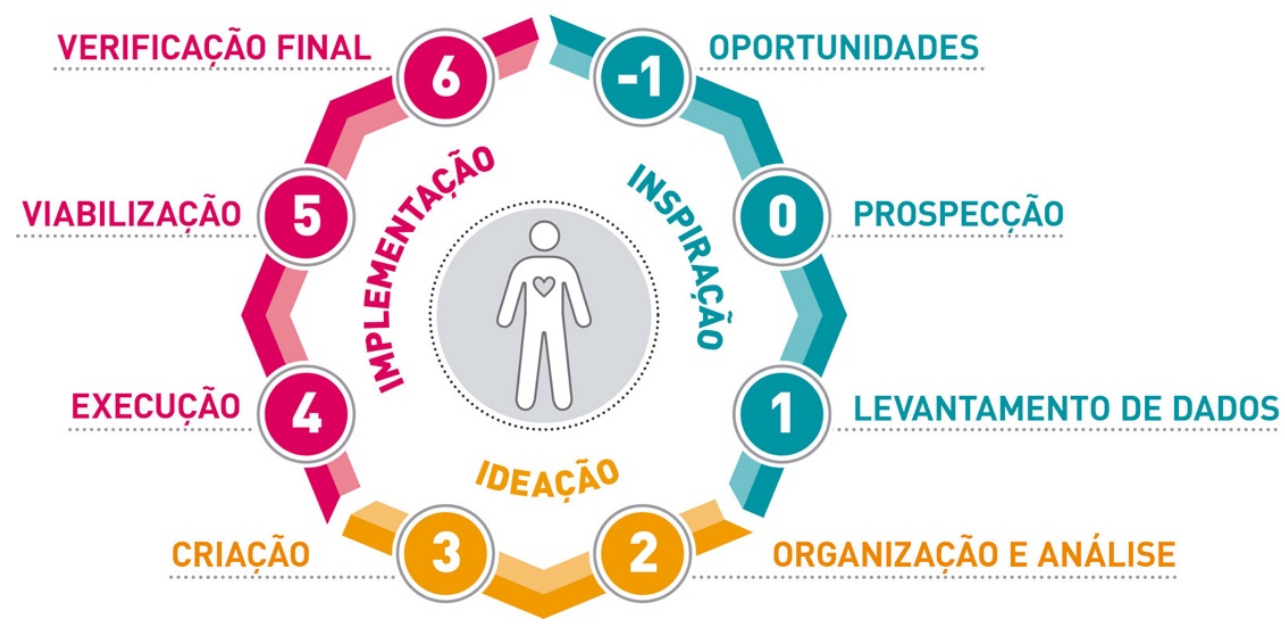

Figura 2 - Representação dos 3 momentos principais e das 8 etapas do GODP. Fonte: Merino (2016).

Devido ao recorte escolhido para essa pesquisa, serão descritas as ações realizadas no momento Inspiração, que compreende as etapas Oportunidades (-1), Prospecção (0) e Levantamento de Dados (1). A descrição dessa pesquisa compreende as ações realizadas entre junho de 2014 a dezembro de 2015. 


\section{ESTUDO DE CASO: A GESTÃO DE DESIGN NO IPQ-SC}

O estudo de caso relatado a seguir compreende ações de gestão de design realizadas no IPq-SC. Inicialmente será apresentado uma breve contextualização e histórico do IPq-SC, objeto desse estudo de caso, e posteriormente serão descritas cada uma das etapas do momento Inspiração da metodologia GODP.

\subsection{IPq-SC}

O IPq-SC, localizado em São José - SC, foi criado em 1941, inicialmente como hospital Colônia Sant' Ana (HCS), com a finalidade de abrigar pacientes psiquiátricos. Em 1971 sofreu um processo de transição, passando a denominação atual - IPq-SC - e abrigando duas novas unidades assistenciais com objetivos distintos: uma delas para internação de pacientes em surto psiquiátrico grave, com proposta de curta permanência, e o Centro de Convivência Santana (CCS) com os leitos dos pacientes remanescentes do antigo hospital (VIEIRA, 2009), e que hoje abriga aproximadamente 220 pacientes asilados moradores. Os pacientes asilados do CSS são portadores de transtornos mentais crônicos e muitos deles possuem vínculo familiar prejudicado ou inexistente, o que dificulta a aplicação das diretrizes da reforma psiquiátrica que prega pela desospitalização e desinstitucionalização desses pacientes, já que a ausência de vínculo familiar dificulta sua reinserção na sociedade.

\subsection{Momento Inspiração (GODP)}

O momento inspiração é o primeiro na metodologia GODP (Figura 2) e se subdivide em 3 etapas: Oportunidades (-1), Prospeç̧ão (0) e Levantamento de Dados (1). A seguir será relatado como foram realizadas cada uma das 3 etapas do momento Inspiração na gestão de design para o projeto realizado no IPq-SC.

\subsubsection{Etapa Oportunidades (-1)}

No segundo semestre do ano de 2014, o NGD/LDU iniciou os contatos com o IPqSC para verificar a oportunidade de desenvolver projetos de pesquisa e extensão com foco no design (gestão de design, design gráfico, design de produtos, design de serviços) e engenharia (ergonomia de produtos e processos) na área da saúde. Em visitas realizadas ao hospital, bem como reuniões com a administração foram identificadas duas linhas de atuação para projetos de design e engenharia, as quais o NGD/LDU possuía capacidade técnica para execução: saúde de pacientes/usuários e saúde dos trabalhadores.

Quanto a capacidade técnica do NGD/LDU, destaca-se que este possui equipe multidisciplinar composta por designers, engenheiros, fisioterapeutas, administradores e médicos, além de possuir equipamentos ${ }^{5}$ necessários para realizar levantamentos e análises no que tange a identificação dos riscos de saúde (ergonômico) e que auxiliam

\footnotetext{
${ }^{5} \mathrm{O}$ NGD/LDU já vem utilizando instrumentação técnica para obter medidas quantitativas para projetos de design e engenharia (CUNHA et al., 2015; SPECK et al., 2016). Os equipamentos do laboratório são: Captura de movimentos (Xsens), Eletromiógrafo de superfície, Dinamômero, Termógrafo digital e rastreamento ocular (Eye Tracking).
} 
nas ações para o desenvolvimento, implementação e principalmente uso de produtos de reabilitação.

Com base na identificação das linhas de atuação e capacidade técnica do NGD/LDU foram procuradas fontes de fomento para realização de projetos de pesquisa e extensão com foco no design e na engenharia. Foram selecionados três editais, para os quais foram enviados projetos:

1) Edital PROEX (MEC-SESu): ações de extensão no IPq-SC com foco em pacientes e trabalhadores;

2) Edital CAPES - RPDTA: pesquisas em nível de pós-graduação integrando design e engenharia mecânica no contexto da Tecnologia Assistiva ${ }^{6}$;

3) Edital Universal CNPq: pesquisas visando o desenvolvimento de produtos em Tecnologia Assistiva.

Os 3 projetos propostos para os editais acima foram aprovados, finalizando a etapa Oportunidades, que pode ser visualizada de maneira resumida na figura 3.

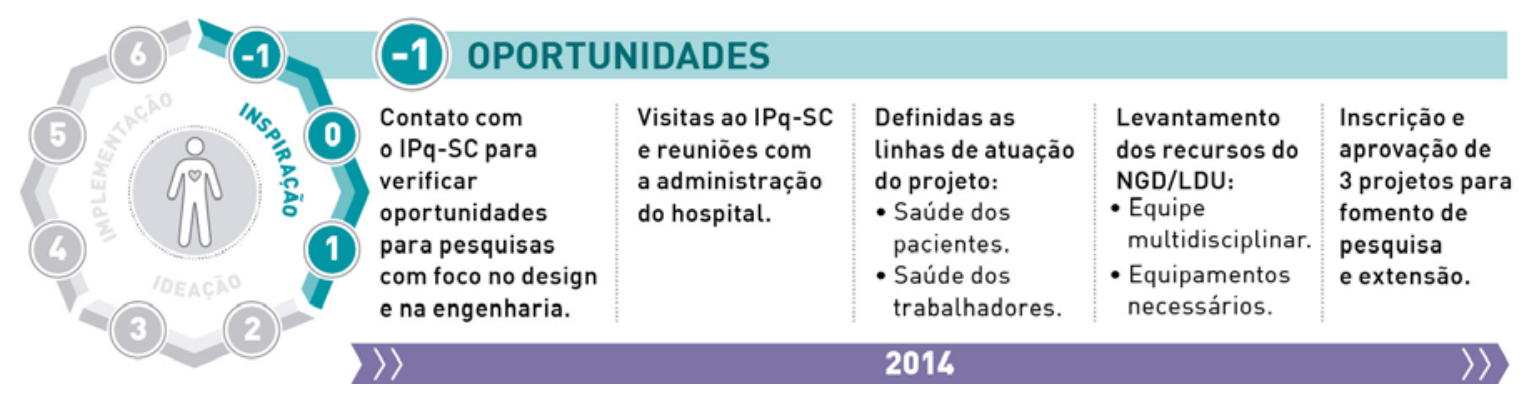

Figura 3 - Resumo da etapa de Oportunidades do GODP.

Fonte: Elaborado pelos autores, com base na pesquisa realizada.

\subsubsection{Etapa Prospecção (0)}

As ações para os 3 projetos foram definidas nessa etapa. Para isso, a equipe de coordenação local (UFSC) reuniu-se com a direção do hospital para entender melhor quais seriam os problemas prioritários do IPq-SC - dentro das linhas de atuação previamente definidas. O contato com a direção do hospital também serviu para que os gestores pudessem entender melhor o papel do design e da engenharia e de que maneira poderiam contribuir para a melhoria dos serviços de saúde.

Foram feitas também diversas visitas no IPq-SC para compreender o contexto em que seriam realizados os projetos. A partir disso, gerou-se um diagrama, que pode ser visualizado na figura 4, e que auxiliou a equipe envolvida na gestão de design a entender as relações entre os atores envolvidos no contexto do IPq-SC.

Também nessa etapa foi feito o cadastro e aprovação do projeto no Comitê de Ética da UFSC (№: 1.257.716), procedimento necessário por tratar-se de uma pesquisa que envolvia seres humanos.

As ações definidas nas duas linhas de atuação previamente selecionadas, que atendiam as demandas prioritárias do IPq-SC, bem como encontravam-se no escopo de atuação do NGD/LDU foram:

\footnotetext{
${ }^{6}$ Tecnologia Assistiva é um termo utilizado para "[...] identificar todo o arsenal de Recursos e Serviços que contribuem para proporcionar ou ampliar habilidades funcionais de pessoas com deficiência e consequentemente promover Vida Independente e Inclusão" (BERSCHE; TONOLLI, 2006).
} 
1) Linha de atuação - pacientes: foco na reabilitação de pacientes asilados moradores, que além dos problemas mentais, possuem limitações impostas pela própria degeneração física causada pela utilização crônica da medicação neuroléptica para o controle da doença. Para isso as ações propostas foram a desenvolvimento de órteses, que ajudariam na sua locomoção e estabilização, além de ações direcionadas a Terapia Ocupacional (TO), buscando o desenvolvimento de equipamentos que auxiliassem na execução das tarefas, que pelas limitações físicas e cognitivas necessitam de equipamentos adaptados.

2) Linha de atuação - trabalhadores: identificação e proposição de soluções para melhorar o ambiente laboral do IPq-SC com foco na farmácia hospitalar e na Terapia Ocupacional.

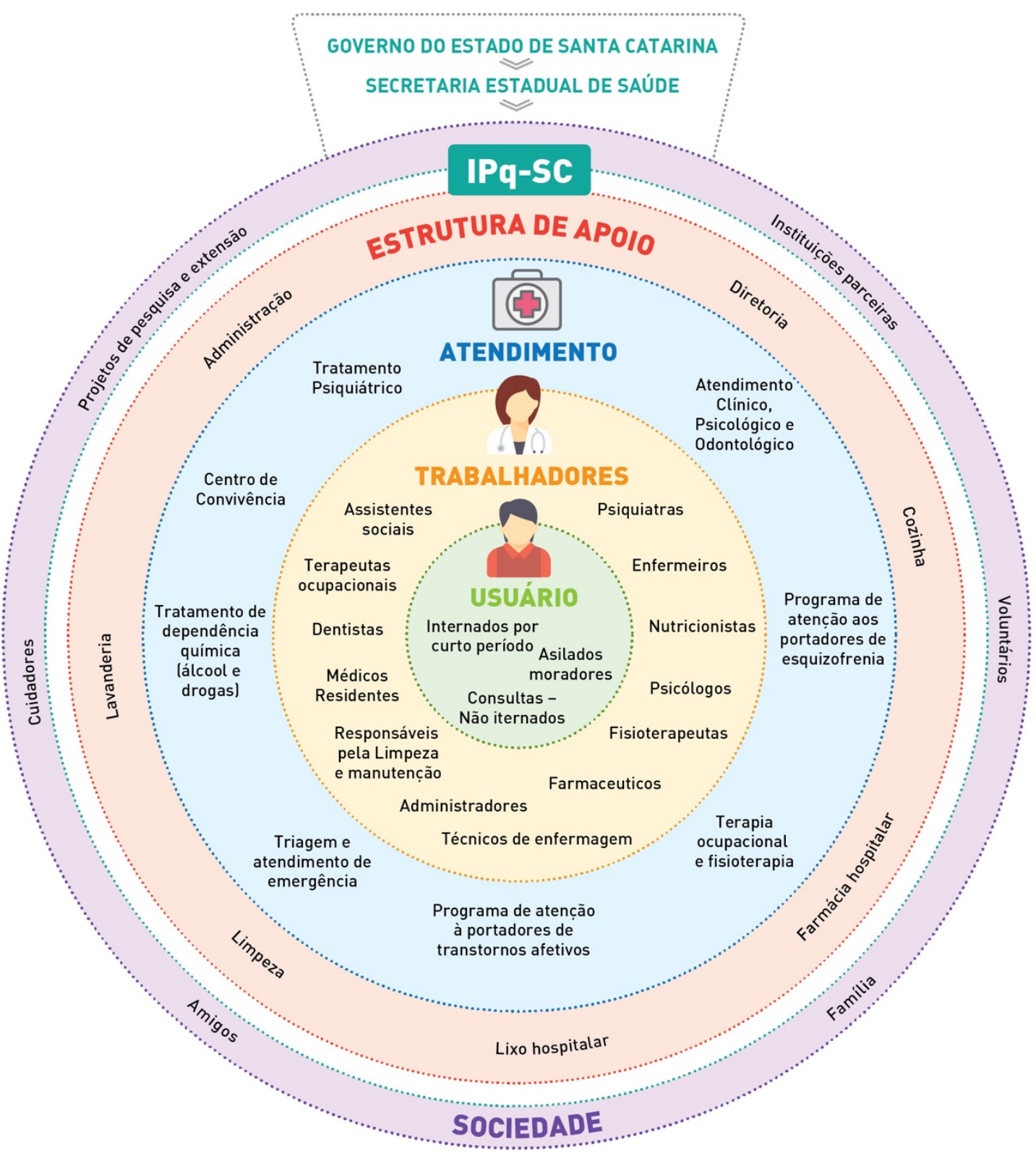

Figura 4 - Diagrama de atores envolvidos no IPq-SC.

Fonte: Elaborado pelos autores, com base na pesquisa realizada. 
Com a definição das ações, se encerrou a etapa de prospecção. O resumo dessa etapa pode ser visualizado na figura 5.

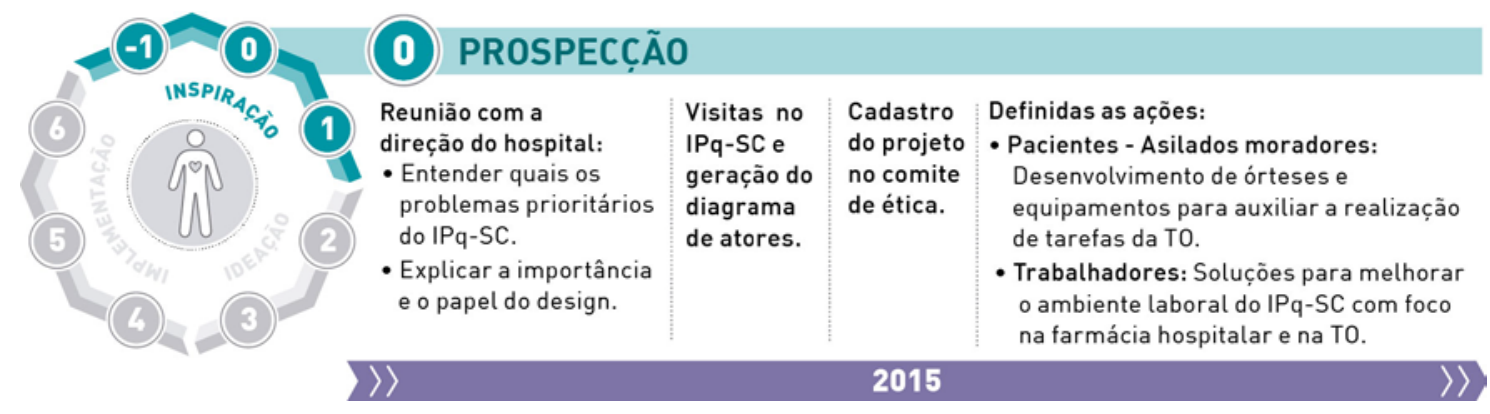

Figura 5 - Resumo da etapa de Prospecção do GODP.

Fonte: Elaborado pelos autores, com base na pesquisa realizada.

\subsubsection{Etapa Levantamento de Dados (1)}

De maneira geral, na etapa de levantamento de dados, foram feitas reuniões com os responsáveis por cada setor (responsáveis, gerentes e diretores). Nessas reuniões, também estavam presentes funcionários que atuavam diretamente nas atividades, como forma de relatar e esclarecer as dificuldades e problemas que existiam em cada um dos setores. Também foram feitas diversas visitas in loco no intuito de compreender o ambiente em que se iria atuar.

Para cada um dos setores foi feita também uma pesquisa bibliográfica para entender melhor o contexto em que se estava trabalhando, bem como procurar problemas e soluções já existentes em contextos similares aos do IPq-SC. Na sequência foram elaborados os blocos de informação para cada uma das ações propostas. Esses blocos de informação auxiliam na visualização das três dimensões que devem ser consideradas para os projetos (produto, contexto e usuário), a figura 6 demonstra como foi feito o bloco de informação para o desenvolvimento de órteses.

\begin{tabular}{|c|c|c|}
\hline PRODUTO & CONTEXTO & USUÁRIO \\
\hline $\begin{array}{l}\text { Órteses para auxilio à } \\
\text { estabilização e locomoção: } \\
\text { - Órteses personalizadas } \\
\text { para cada paciente. } \\
\text { - Materiais de baixo custo. } \\
\text { - Órteses para membros } \\
\text { inferiores (auxiliar na } \\
\text { marcha e estabilização). }\end{array}$ & $\begin{array}{l}\text { IPq-SC - Centro de } \\
\text { Convivência Santana (CCS): } \\
\text { - Abriga pacientes asilados } \\
\text { moradores. } \\
\text { - Muitos desses pacientes } \\
\text { perderam o vínculo familiar. } \\
\text { - Possui fisioterapeutas para } \\
\text { auxiliar os pacientes com } \\
\text { dificuldade de locomoção. } \\
\text { - Sobrecarga física dos } \\
\text { fisioterapeutas para ajudar } \\
\text { os pacientes na locomoção. }\end{array}$ & $\begin{array}{l}\text { Pacientes asilados } \\
\text { moradores: } \\
\text { - Problemas mentais. } \\
\text { - Degeneração física causada } \\
\text { pela utilização crônica da } \\
\text { medicação neuroléptica } \\
\text { para o controle da doença. } \\
\text { - Dificuldade de locomoção. } \\
\text { - Dificuldade em se manter } \\
\text { de pé. }\end{array}$ \\
\hline
\end{tabular}

Figura 6 - Bloco de informação para as Órteses.

Fonte: Elaborado pelos autores, com base na pesquisa realizada.

Observou-se a partir do contexto presente no bloco de informação das órteses, que o desenvolvimento desse projeto poderia beneficiar não somente o usuário 
(pacientes asilados moradores), mas também auxiliaria os trabalhadores, principalmente os fisioterapeutas, que se sentiam sobrecarregados quando necessitavam ajudar o paciente tanto na sua locomoção, como na própria estabilização. A partir da coleta das informações e melhor conhecimento de cada uma das áreas de atuação, foram definidas sub-ações para cada uma das ações propostas. Nesse momento, o GODP foi aplicado para cada uma das ações propostas, sendo assim, o momento inspiração inicia-se novamente, mas com ações direcionadas. Como exemplo da ação das órteses, foram feitas análises morfológicas, sincrônicas e diacrônicas, além de pesquisa bibliográfica e levantamento do perfil de cada um dos pacientes para os quais seriam desenvolvidas órteses. No entanto, como o intuito dessa pesquisa é demonstrar um panorama geral da gestão de design de todo o projeto, que compreende inicialmente as três etapas listadas anteriormente (Oportunidade, Prospecção e Levantamento de Dados), não foi possível detalhar cada uma das ações. O resumo do que foi feito na etapa levantamento de dados pode ser visualizado na figura 7.

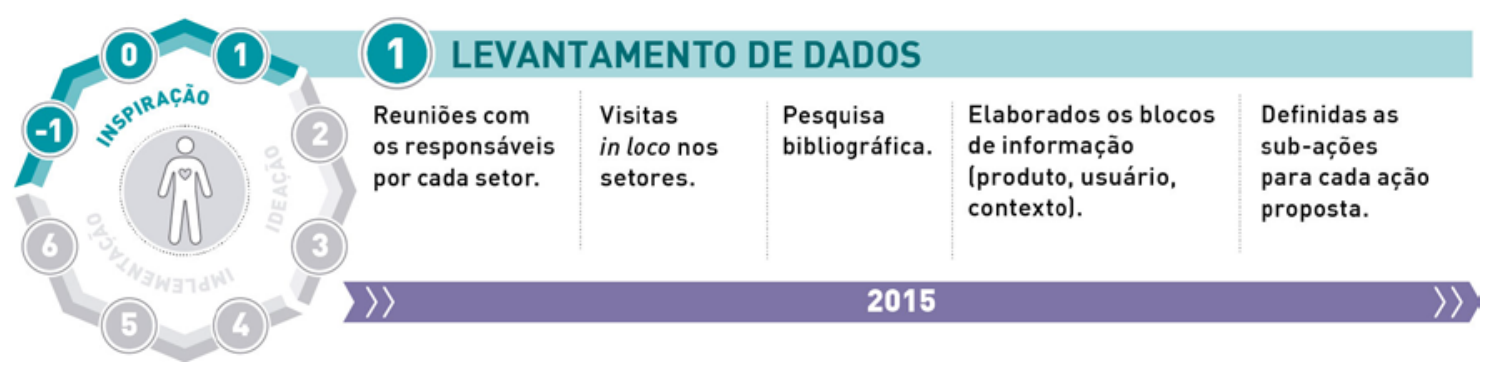

Figura 7 - Resumo da etapa de Levantamento de Dados do GODP.

Fonte: Elaborado pelos autores, com base na pesquisa realizada.

\section{CONSIDERAÇÕES FINAIS}

O estudo de caso apresentado compreende as primeiras etapas de um processo de gestão de design feito no IPq-SC. Trata-se da soma de 3 projetos que se encontram em execução, e que a partir do recorte apresentado nessa pesquisa, foi possível evidenciar a complexidade envolvida na gestão de design e a necessidade de coordenar as ações dos projetos de acordo com os objetivos da organização a partir de uma visão holística, considerando o contexto onde o design está inserido.

Destaca-se que a pesquisa englobou a atuação de diversos profissionais como designers, engenheiros, fisioterapeutas, enfermeiros, médicos e administradores. Foi importante nesse caso, estabelecer uma linguagem comum a todos os profissionais envolvidos, transformando a linguagem técnica de cada área para que o conhecimento pudesse ser partilhado por todos os profissionais. O uso de figuras, como foi demonstrado ao longo da pesquisa apresentada ajudou na uniformização dessa linguagem. Também é importante destacar que o design não está sozinho buscando soluções, mas que depende de diversas áreas que devem atuar de maneira conjunta, unindo competências e conhecimentos (ROSA; ROMANO; BATTISTEL, 2014).

Os projetos relatados podem ser colocados como as primeiras iniciativas de inserção do design no IPq-SC e por isso, foram feitos de maneira gradativa e responsável. A sensibilização da diretoria do hospital foi importante para que o papel do design e da engenharia fosse compreendido no IPq-SC. Foi a partir da aceitação e 
da confiança estabelecida entre a equipe e o diretor do hospital, que a equipe do projeto pode contar com a colaboração dos demais trabalhadores do hospital. Essa relação de confiança torna-se necessária, já que o projetista necessita saber das necessidades e do contexto no qual irá projetar e para isso foi essencial a colaboração dos profissionais do IPq-SC.

O estudo de caso apresentado ajudou a evidenciar as contribuições que a gestão de design pode oferecer para que projetos de design, envolvendo outras áreas de atuação - como engenharia, fisioterapia, etc. - possam contribuir para a melhoria dos serviços de saúde em um hospital psiquiátrico.

A pesquisa apresentada representa um recorte dos projetos desenvolvidos pelo NGD/LDU no IPq-SC. Destaca-se que além das ações apresentadas nessa pesquisa, existem ainda outras ações em desenvolvimento, e que englobam outros setores do hospital como na psicologia (convivência de pacientes) e enfermagem.

\section{AGRADECIMENTOS}

Os autores agradecem a CAPES, ao CNPq, a FAPEU, a RPDTA, ao IPq-SC e ao NGD/LDU que viabilizaram esta pesquisa.

\section{REFERÊNCIAS}

AVENDAÑO, Luis Emiliano Costa. Resgate do protagonismo do desenhista industrial por meio da gestão do design. Pós. Rev. Programa Pós-grad. Arquit. Urban.

Fauusp, [s.l.], n. 17, p.34-45, 1 jun. 2005. Universidade de Sao Paulo Sistema Integrado de Bibliotecas - SIBiUSP. http://dx.doi.org/10.11606/issn.2317-2762.v0i17p34-45.

BERSCHE, Rita; TONOLLI, José Carlos. Introdução ao Conceito de Tecnologia Assistiva e Modelos de Abordagem da Deficiência. 2006. Disponível em: <http://www.bengala legal.com/tecnologia-assistiva>. Acesso em: 20 maio 2016.

BEST, Kathryn. Fundamentos de Gestão de Design. Porto Alegre: Bookman, 2012.

BRASIL. Ministério da Saúde. Secretaria de Assistência à Saúde (Org.). Programa Nacional de Humanização da Assistência Hospitalar. Brasília: Ministério da Saúde, $2001 a$

BRASIL. Presidência da República. Casa Civil. Subchefia para Assuntos Jurídicos. Lei no 10.216, de 06 de abril de 2001. Dispõe sobre a proteção e os direitos das pessoas portadoras de transtornos mentais e redireciona o modelo assistencial em saúde mental. Brasília, 2001b. Disponível em: <http://www.planalto.gov.br/ccivil_03/ Leis/LEIS_2001/L10216.htm>. Acesso em: 20 mai. 2016.

CENTRO PORTUGUÊS DE DESIGN (Portugal). Manual de Gestão de Design. Porto: Porto Editora, 1997.

CUNHA, Julia Marina et al. O uso de sensores inerciais (Xsens) no projeto de produto aplicado a ferramenta agrícola. In: INTERNATIONAL CONFERENCE ON INTEGRATION OF DESIGN, ENGINEERING AND MANAGEMENT FOR INNOVATION., 4., 2015, Florianópolis. Anais... . Florianópolis: Udesc, 2015. p. 1 - 14. Disponível em: <http://limonadadesign.com.br/idemi2015/anais/03/142762.pdf>. Acesso em: 24 maio 2016. 
DMI - DESIGN MANAGEMENT INSTITUTE. What is Design Management? 2016. Disponível em: <http://www.dmi.org/?What_is_Design_Manag>. Acesso em: 01 maio 2016.

GIL, Antonio Carlos. Métodos e Técnicas de Pesquisa Social. 5. ed. São Paulo: Atlas, 2007.

JONES, Peter. Design for Care: Innovating Healthcare Experience. New York: Rosenfeld, 2013.

LIMA JÚNIOR, José Humberto Viana; ÉSTHER, Angelo Brigato. Transições, prazer e dor no trabalho de enfermagem. Revista de Administração de Empresas, São Paulo, v. 41, n. 3, p.20-30, set. 2001. Disponível em: <http://www.scielo.br/pdf/rae/v41n3/ v41n3a03.pdf>. Acesso em: 23 ago. 2016.

LUCIO, C. C; PASCHOARELLI, L. C. Usabilidade e acessibilidade de equipamentos médico-hospitalares: um estudo de caso com pacientes obesos. In: PASCHOARELLI, L. C.; MENEZES, M. dos S. (Orgs). Design e ergonomia: aspectos tecnológicos. São Paulo: Cultura Acadêmica, 2009.

MACIEL, Silvana Carneiro. Reforma psiquiátrica no Brasil: algumas reflexões.

Cad. Bras. Saúde Mental, Rio de Janeiro, v. 4, n. 8, p. 73-82, jun. 2012.

MARTINS, Rosane Fonseca de F.; MERINO, Eugenio Andrés Diaz. A Gestão de Design como estratégia organizacional. 2. ed. Rio de Janeiro: Rio Books, 2011.

MERINO, Giselle Schmidt Alves Díaz. Metodologia para a prática projetual do Design com base no Projeto Centrado no Usuário e com ênfase no Design Universal. 2014. 212p. Tese (doutorado). Universidade Federal de Santa Catarina, Curso de Pósgraduação em Engenharia de Produção.

MERINO, Giselle Schmidt Alves Díaz. GODP - Guia de orientação para o desenvolvimento de projetos: uma metodologia centrada no usuário. Florianópolis: UFSC/NGD-LDU, 2016.

MOREIRA, Bruna Ruschel et al. Gestão do Design na prática: Framework para implementação em empresas. Porto Alegre: Marca Visual, 2016. 145 p. Disponível em: <http://www.ufrgs.br/icd>. Acesso em: 1 maio 2016.

MOZOTA, Brigitte Borja de. Design Management: Using Design to Build Brand Value and Corporate Innovation. New York: Allworth Press, 2003.

NGD/LDU - NÚCLEO DE GESTÃO DE DESIGN \& LABORATÓRIO DE DESIGN E USABILIDADE (Florianópolis). Universidade Federal de Santa Catarina. Apresentação. 2016. Disponível em: <http://www.ngd.ufsc.br/apresentacao/>. Acesso em: 25 abr. 2016.

PARK, June H.. Health Care Design: Current and Potential Research and Development. Design Issues, [s.I.], v. 31, n. 1, p.63-72, jan. 2015. MIT Press - Journals. http://dx.doi.org/10.1162/desi_a_00310.

PRESIDÊNCIA DA REPÚBLICA. Constituição (2011). Decreto no 7602, de 7 de novembro de 2011. Política Nacional de SeguranÇa e SaÚde no Trabalho. Disponível em: <http://www.planalto.gov.br/ccivil_03/_Ato2011-2014/2011/Decreto/D7602. htm>. Acesso em: 23 maio 2016.

REID, Proctor P.; COMPTON, W. Dale; GROSMANN, Jerome H.; FANJIANG, Gary. Building a better delivery system: a new engineering/health care 
partnership. The National Academy Press. Washington, 2005.

ROSA, Catherine Ribeiro; ROMANO, Fabiane Vieira; BATTISTEL, Amara Holanda. Design no ambiente hospitalar: mesa para múltiplas atividades. In: CONGRESSO BRASILEIRO DE PESQUISA E DESENVOLVIMENTO EM DESIGN, 11., 2014, Gramado. Proceedings... . Gramado: Blucher Design Proceedings, 2014. v. 1, n. 4, p. 1 - 12. Disponível em: <www.proceedings.blucher.com.br/evento/11ped>. Acesso em: 23 maio 2016.

SERRANHEIRA, Florentino; UVA, António de Souza S.; SOUSA, Paulo. Ergonomia hospitalar e segurança do doente: mais convergências que divergências. Revista Portuguesa de Saúde Pública, v. 10, p. 58-73, 2010.

SILVA, Edna Lúcia; MENEZES, Estera Muszkat. Metodologia da pesquisa e elaboração de dissertação. 4. ed. Florianópolis: Laboratório de Ensino a Distância da UFSC, 2005.

SPECK, Giselle Mari et al. Processo de instrumentação integrada no desenvolvimento de projetos de tecnologia assistiva. In: CONGRESSO BRASILEIRO DE ERGONOMIA, 18., 2016, Belo Horizonte. Anais... . Belo Horizonte: Ufmg, 2016. p. 1 - 7.

STROPKAY, Scott; SIEDZIK, David. Embracing Complexity: A Framework for Healthcare Design. Design Management Institute, [s.i], p.42-48, primavera 2013.

WHO - World Health Organization. Health. 2016. Disponível em: <http://www.who. int/trade/glossary/story046/en/>. Acesso em: 20 mai. $2016 a$.

WHO - World Health Organization. Hospitals. 2016. Disponível em: < http://www.who.int/topics/hospitals/en/>. Acesso em: 20 mai. 2016b.

VIEIRA, Raquel dos Santos. $O$ serviço social no atendimento as famílias dos usuários internados na unidade de dependência química do Instituto de Psiquiatria do Estado de Santa Catarina. 2009. 91 f. TCC (Graduação) - Curso de Serviço Social, Universidade do Sul de Santa Catarina, Palhoça, 2009. 